\title{
An Investigation on IoT Healthcare Analytics
}

\author{
Shanmugasundaram. $\mathbf{G}^{\mathbf{1}}$ \\ Department of Information Technology, Sri Manakula Vinayagar Engineering College, Pondicherry, India. \\ Email: shanmugasundaram@smvec.ac.in
}

Sankarikaarguzhali.G ${ }^{2}$

PG Student, Department of Information Technology, Sri Manakula Vinayagar Engineering College, Pondicherry, India. Email: gsankari2012@gmail.com

\begin{abstract}
The Internet of Things makes things to become active participants enabling communication between things and transfer of data among themselves. It has a wide range of applications in various domain such as healthcare. The Internet of Things changes the way of delivering healthcare solution to people. It maintains a smooth relationship between doctors and patients which leads to high quality results in medical care. These are achieved by continuous monitoring of patients by using sensors. The data collected are recorded and used for analytics in future purpose. The analytical solution in healthcare data gives a potential for identification of diseases. This paper concerns the Internet of Things in healthcare and also portrays the technology involved in it. The paper discuss the architectures and services involved in medical care solution. Further the mechanism involved in healthcare analytics and data sources involved in analytics are explained. The various algorithms involved in it are investigated. It also analyses the various challenges in healthcare perspective.
\end{abstract}

Index Terms - Internet of Things, Healthcare, Analytics

\section{INTRODUCTION}

Internet of Things is a recent technology that intends to upgrade the different forms of communication we have at present. IoT enables the interconnection of uniquely identifiable things using internet providing world with extended benefits [1].In general Internet of Things is defined as the interconnection of physical objects through internet which provide status to humans and allowing them to control from remote places with the help of internet. The role of IoT in smart healthcare is important on the aim of providing better healthcare services and improve the quality of life of citizens. This is achieved by continuous monitoring of persons physical condition and rendering them by automated processing and storage of information. The healthcare services are provided by using various technologies like identification technology, communication technology, sensing technology, location technology and visualization. By using these technologies IoT architecture are formed. There is no particular architecture exist for IoT. Several architectures exists like healthcare architecture, three layer architecture for smart grids, stratecast architecture for smart home etc.

Better treatments and technologies involved in healthcare is achieved by Analytics in it. Databases and servers plays a vital role in creating health record which is used for analytics providing on-demand health services [4].Analysis is done by considering various data sources. The analytics uses various machine learning algorithm for identifying the diseases. Machine learning algorithms are self learning algorithms which increases the accuracy of diagnosis by predictive analysis. Support vector machine is a machine learning algorithm which plays a vital role in classifying data and identifying the diseases in an effective manner.

The structure of the paper is as follows: Section 2 is focused on technologies in healthcare involving identification technology, communication technology, sensing technology, location technology and visualization. Content 3 introduces IoT architecture in the field of healthcare. Part 4 contributes to the introduction of healthcare analytics. Section 5 contain healthcare challengesand section 6 deals with healthcare analytics and content 7 contains data sources.Part 8 provides the machine learning algorithms.Discussion is done at section 9 .

\section{TeChnOlOGIES IN HeAlthCARE}

IoT based healthcare system forms a network by interconnecting the devices to perform healthcare activities such as monitoring, diagnosis of diseases , and also perform remote surgeries using internet. Many clinical grade wearable's are now available to track patients data. Nowadays sensor networks are used within hospitals and practices for providing healthcare delivery. Presently, the report says that Healthcare Internet of Things is steady to hit $\$ 117$ billion by 2020 [15].

Internet of Things in healthcare is made possible by integration of several technologies. The following are the enabling technologies in healthcare.

\section{A. Identification Technology}

A practical IoT includes large number of devices each of which generates data and authorized person can access data no matter where is located. A device is provided with unique identifier(UID). Hospital, Rehabilitation center, doctor or nurse is associated with a digital UID. 
The relation between them is provided by the digital domain which allow things in the network to be identified specifically. Standards like Universally Unique Identifier (UUID) and Globally Unique Identifier (GUID) [5]. For identification of objects IoT uses technology namely RFID. Radio Frequency Identification is a automated identification technique which operate wirelessly to identify the object and obtain the content. The necessary components in RFID system are electronic label, a reader and supervising computer. The electronic label is a radio frequency card or noncontact IC card (also referred to as a tag). It consists of an antenna and label chip with circuitry within it, which provides control and storage. There are two types of electronic labels namely passive and active. In healthcare a passive RFID is used to keep track of people's medical records and acquire information about people. A passive RFID doesn't have a power supply from the inductive reader [7].

\section{B. Sensing technology}

The physiological paramaters of a person are tracked continuously by using sensing technology. These parameters are used by the doctors for the diagnosis of diseases[13]. It facilitates better treatment for patients and reduces the costs involved in healthcare. IoT healthcare system uses devices like pulse oximeter, motion analysis sensor, tri-axial accelerometer, heart beat sensor etc. Here some of the IoT devices which operates based on sensing technology are discussed. Pulse oximeter are used to obtain the heart rate and blood oxygen saturation which act as sign for emergency service. It is also used for disease diagnosis[1]. Cerebral Pressure sensor are used to identify the pressure changes in brain. The sensor is operated wirelessly. It is used to identify a disease called dementia which is caused by change in cerebral pressure. Motion analysis sensor encapsulates many sensors embedded within it. It is used to record the patients motion continuously. A tri-axial accelerometer detects the orientation and movement of each part of the body.

\section{Communication Technology}

Communication technology is used to transfer of information over networks and it forms an IoT healthcare subsystem. The data collected by sensors are transmitted over the networks using various wireless technologies like Zigbee, Wi-Fi, Infrared data association etc. These technologies has different radio frequency, security standards, transmission rate etc.

Bluetooth is a technology used for medical environment due to its low radiation characteristic and harmless to human. It is a wireless technology standard for exchanging data over shorter distance. It operates at a frequency of $2.4 \mathrm{GHZ}$ and over a range of 1 to $100 \mathrm{~m}$. The technology is inexpensive and it eliminates the need for cabling[12]. A set of Bluetooth devices sharing a common channel forms a piconet. Bluetooth uses a specification of IEEE 802.15.1 Standard. The new Bluetooth Low-Energy (BLE) or Bluetooth Smart, as it is now branded as a significant protocol for IoT applications.

Zigbee is used mostly for collecting medical data and transferring them. It plays an important role in IoT healthcare applications. It also forms wireless sensor technology specifically for low rate sensors. It consists of four layers namely Physical, MAC, Network layer and Application layer [13]. The physical and MAC layers are based on IEEE 802.15.4 standard. In Network layer Zigbee contains 3 device types namely, Zigbee router, Zigbee coordinator and End device. ZigBee operates around the range of around 100 meters and a bandwidth of $250 \mathrm{kbps}$.Star, cluster,tree and mesh are the topologies it uses. Zigbee is widely used in home automation, intelligence agriculture, industrial discipline, medical monitoring \& power systems [7].

Wi-Fi is the most popular technology for medical applications. Wireless fidelity is a networking technology that enables computers and devices to communicate over a wireless signal. It is based on 802.11n standard .It operates on $2.4 \mathrm{GHZ}$ and $5 \mathrm{GHZ}$ frequency bands and approximately in the range of more than $100 \mathrm{~m}$. It operates at a data rate of $150-200 \mathrm{Mbps}$. Wi-Fi is used to connect to cloud easily[22]. IoT enables a borderless world where things speak to the cloud.

A WSN can generally be described as a cluster of nodes that cooperatively sense data and it helps to control the environment, enabling the intercommunication between persons or objects and the $y$ can be used in IoT paradigm.

Sensor nodes guide the collected data to transmit along other sensor nodes by using hopping technique. At the time of transmission, monitored data may pass through multiple nodes to get to gateway node after multihop routing, and finally reach the end node using the internet or satellite. It is the user who configures and guides the WSN with the management node, report monitoring missions and collection of the monitored data [24]. 


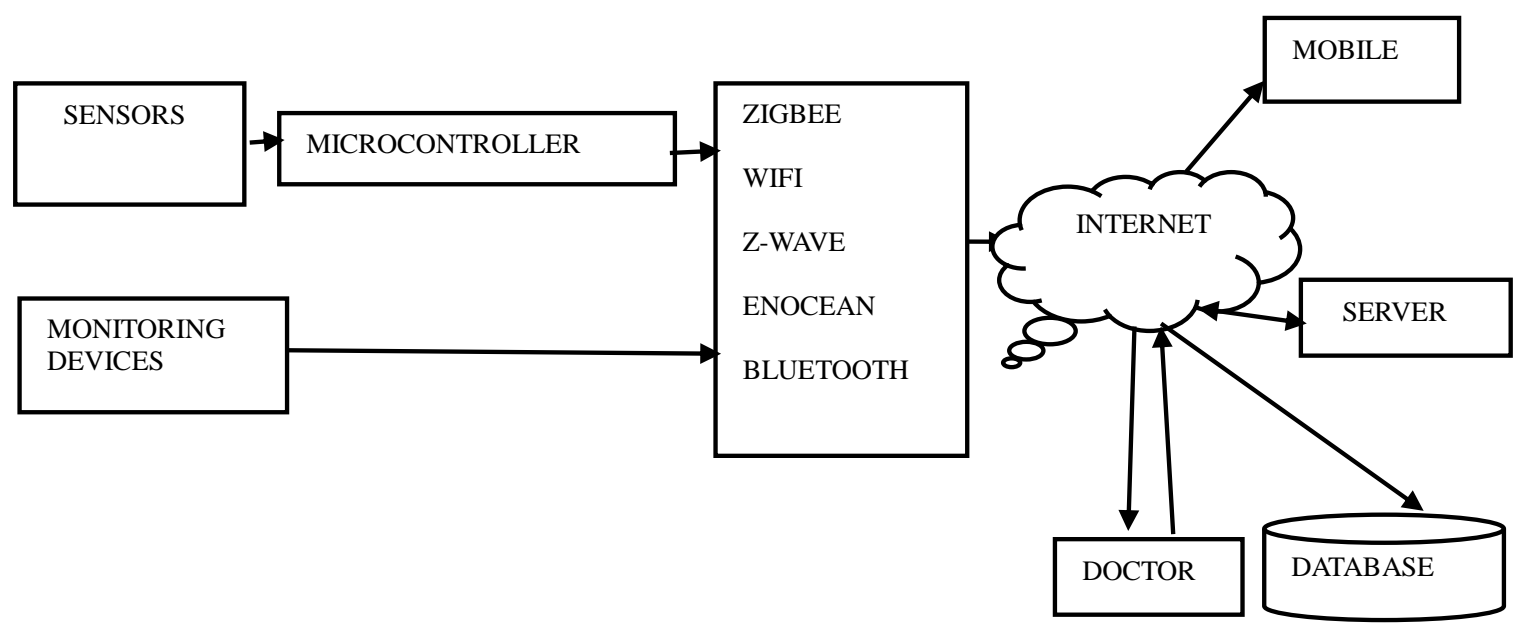

Fig.1. IoT Healthcare System.

\section{Location Technology}

Real-time location systems (RTLS) are used to track the locations of people. It also tracks the treatment process with security, and helps to rearrange the health care system remotely according to location of patient [13]. The most used RTLS is the Global Positioning System (GPS), which operates using satellite-based navigation system. It locate objects under all weather conditions. In healthcare GPS are be used to locate ambulances, patients, doctors, etc.

Local positioning system (LPS) is used to combination with GPS for providing location accuracy. It uses radio signals which travel between objects and a receiver [1].

\section{E. Visualization}

Visualization in IoT is demanding because it enables interaction of users with the environment. The recent technology leads to the use of tablets, smart phones, laptops etc. that has become spontaneous. The data collected by the sensors are transmitted over the network using various communication technologies. There exists various visualization tools like Think Speak ,Freeboard, IBM Blue mix etc.[20].

\section{ARCHITECTURE IN HEALTHCARE}

The architecture of IoT differs for various application present in it. There is no general architecture present for Internet of Things. Based on the technologies involved IoT healthcare architecture is developed.Fig. 1 shows the IoT healthcare architecture. The physiological conditions of the people are continuously sensed by sensors and monitoring devices are also used. The data obtained are then passed over the internet. Wireless networks like Zigbee, Wi-Fi, Z-Wave, EnOcean, Bluetooth etc. are used for transmission based on the range to be transmitted. The data transmitted is received at the server. They get stored in the database automatically. Measured value is also sent to the doctor clinic and mobile phone of people. Feedback by the doctor was also sent to mobile phone of people. People can access their data from the database directly. It also contains information about various doctors and the patient can select the doctors by considering various factors for disease diagnosis. Doctors can also use the database for checking the status of the patient's health.

\section{IOT SERVICE ARCHITECTURE}

The healthcare architecture shown contains three layers namely, information perception layer, network transmission layer and application service layer.

Information perception layer involves the presence of sensors. Sensors are used to continuously monitor the people. It is used to collect physical conditions of the patients[1]. The data's collected are transferred over the network and the data's are stored in database via internet. For transmission of data wireless technologies like Wi-Fi, Zigbee, Enocean etc. are used. Application service layer combines the IOT and medical health service center leading a remote health care service. Some of the healthcare services are discussed here [18].

\section{A. Glucose Level Monitoring}

Most of the people in our country are affected by diabetes. It needs continuous monitoring of glucose level. An m-IoT monitoring provides non-invasive glucose monitoring. Here patients are monitored through sensors and are connected through network of IPV6 to significant healthcare providers. The device includes blood glucose collector, mobile phone and a IoT based medical acquisition detector to monitor the glucose level[9]. Monitoring of glucose level helps in monitoring individual patterns of modification in glucose level leading to plan for meals, activities, medication times etc.

\section{B. Electrocardiogram Monitoring}

The heart rate and basic rhythm are measured as well as the identification of multifaceted arrhythmias, 
Information perception layer

Application service layer

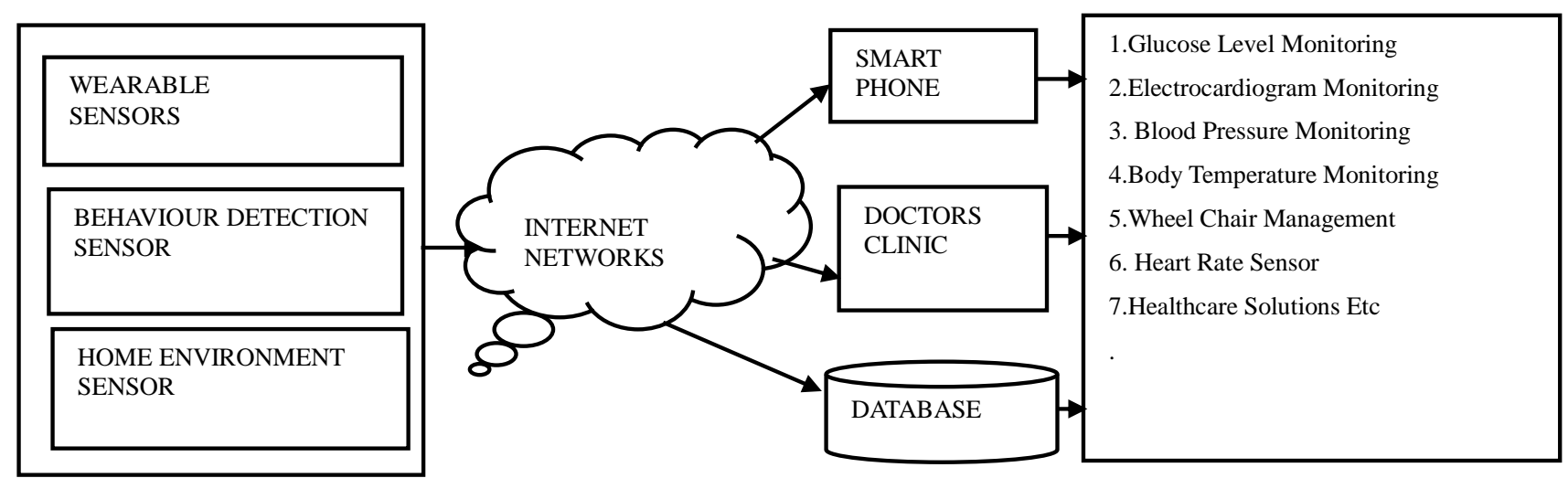

Fig.2. IoT service architecture.

myocardial ischemia, and prolonged QT intervals are done using electrocardiogram monitoring. It is done by recording the electrical activity of the heart. It has the widest application leading to diagnosis of various diseases.

The ECG system comprises of transmitter and receiver which acts wirelessly. It also contains automated method to identify the abnormal activity of heart and they are transferred in real time basis to mobile phones and doctor's clinic over networks. IoT application uses algorithms for ECG monitoring continuously [1].

\section{Blood Pressure Monitoring}

A wearable sensor can be used for monitoring of blood pressure. The device is made up of $\mathrm{BP}$ apparatus with communication module embedded it. Blipcare is a Wi-Fi based blood pressure monitoring device which uses home Wi-Fi for simply uploading its recorded value. These devices contains LCD display which shows the immediate value. An apparatus for blood pressure monitoring was proposed in [21].

\section{Body Temperature Monitoring}

Homeostasis can be identified by change in body temperature. Thus it act as a essential part in providing healthcare services. The m-IoT concept is attained by using body temperature sensor embedded in a TelosB mote. The temperature measurement using home gateway through IoT was proposed in [22]. The body temperature was transmitted by using infrared detection by the home gateway. The main components in the system includes RFID module and body temperature monitoring device.

\section{E. Wheel Chair Management}

Many researches have done on smart wheelchair based on IoT for disabled people. An healthcare system for disabled people based on IoT technology was proposed in [23]. The system consists of various sensors and also uses WBANs technology. The system gives chair vibration control and also detects the status of the wheel chair user. The device monitors the individual's sitting position and also provides information about the surroundings.

\section{F. Heart Rate Sensor}

Nowadays, smartphone acts as the main driver of IoT. The electronic device now operated based on smartphone controlled sensor. Smart phone acts as a versatile healthcare device and the hardware and software products developed for it[18]. The smartphone is provided with healthcare applications which includes general as well as medical education, information search apps etc. Nowadays noncontact measurements for healthcare applications are developed which operates based on image analysis algorithm. Diseases like asthma, chronic obstructive pulmonary disease, cystic broses, coughing, allergic rhinitis, nose-related symptoms of the respiratory tract, the heart rate, $\mathrm{BP}$, blood oxygen saturation etc., can be effectively identified using smart phone

\section{G. Healthcare Solution}

Nowadays, smartphone acts as the main driver of IoT. The electronic device now operated based on smartphone controlled sensor. Smart phone acts as a versatile healthcare device and the hardware and software products developed for it. The smartphone is provided with healthcare applications which includes general as well as medical education, information search apps etc. Nowadays noncontact measurements for healthcare applications are developed which operates based on image analysis algorithm[19]. Diseases like asthma, chronic obstructive pulmonary disease, cystic broses, coughing, allergic rhinitis, nose-related symptoms of the respiratory tract, the heart rate, $\mathrm{BP}$, blood oxygen saturation etc., can be effectively identified using smart phone.

\section{IOT HEALTHCARE CHALLENGES}

The IoT healthcare is growing rapidly. The medical sector is getting adopted to IoT devices and applications forming e-health. The devices deals with people private 
healthcare data. At the same time the devices are connected to networks to obtain data at any time and anywhere. And therefore this domain becomes the target for attackers. To adopt IoT in healthcare domain completely it must be provided with security and privacy. Challenges present in IoT healthcare services includes the following:

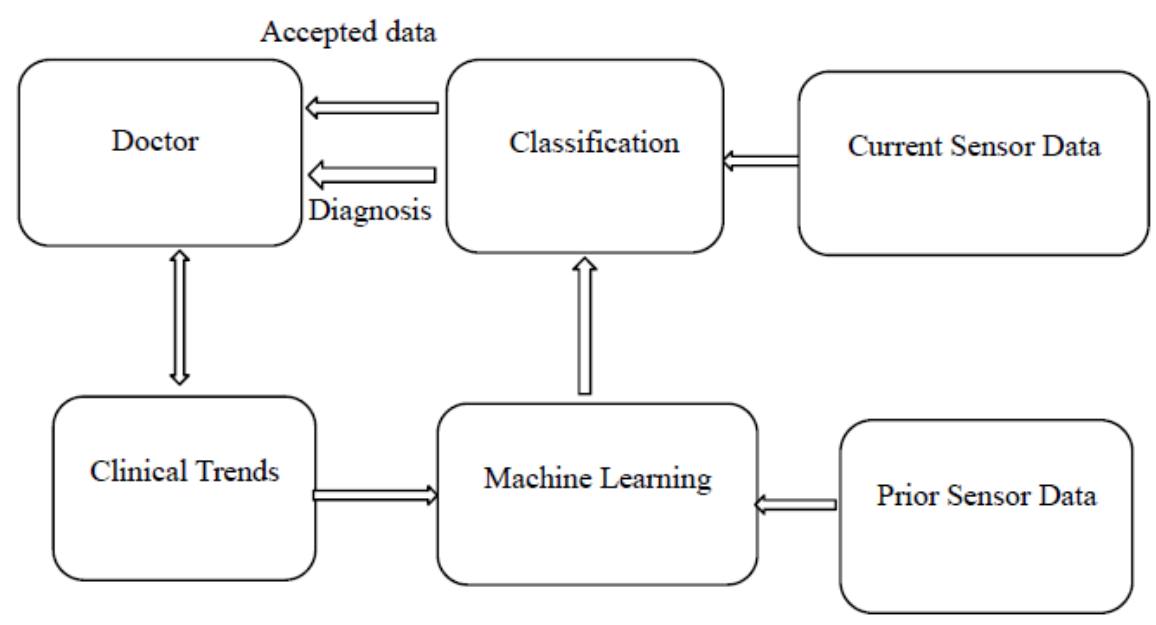

Fig.3. Analytics Workflow For Wearable Sensor Technology System.

\section{Scalability:}

Billions of IoT devices are connected to the network which makes large amount of data need to be processed and stored.[9] The system must be scalable for storing the information obtained from IoT devices. The current data uses Big Data and Cloud for storing the information.

\section{Interoperability:}

In healthcare industry there are many manufacturers giving different product and devices. They have not followed any standard protocols across the devices. This leads to interoperability issues[18]. The device diversity and management of value added services are the major standardization issues present.

Safety:

In many situations people needs continuous monitoring which uses a wearable sensors throughout. In those case any breaches in security becomes life threatening and critical[2].

\section{Security:}

The protection of data obtained from devices and sensors is essential. It requires policies and technical security measures to share data with organizations and authorized users [3]. IoT Healthcare must be ensured with confidentiality, Integrity and availability of people's personal data.

Resource Efficient Security: IoT healthcare needs minimum use of resource with maximum security performance.

Physical Security: The healthcare devices needs Tampering resistant packaging[3]. Attackers may capture the health devices and obtain the secrets of device and alter the program for obtaining the data.

Secure Routing: Devices are susceptible to network attacks. It need routing protocol for secure transmission of data [20].

\section{Data Transparency:}

IoT medical devices are provided with cloud services[2]. The services must be in such a way the patient's data must be tracked and data use must be controlled.

\section{Mobility:}

The IoT healthcare network must allow the mobility of patients and must able to connect anywhere at anytime. This feature enable to connect dissimilar patient environment [20].

\section{Network Type:}

It becomes an open issue to find the type of network. There are three types of networks namely data centric, service centric and patient centric architectures. Healthcare structures are separated based on captured health data called data centric network[4]. Structures formed by assembly of services they provide called service centric. Structures formed by involvement of people for treatment are called patient centric architecture.

\section{IOT IN HEALTHCARE ANALYTICS}

The analysis of data is done by maintaining electronic health records, diagnostic information gathered from imaging equipment upgrades the professionals with decision making powers and enabling the patients to take care of their health. The analytics helps to prevent patient suffering from the chronicle diseases[7] .The analysis of data is done by maintaining electronic health records ,diagnostic information gathered from imaging 
equipment upgrades the professionals with decision making powers and enabling the patients to take care of their health.

The analysis of data is done by maintaining electronic health records, diagnostic information gathered from imaging equipment upgrades the professionals with decision making powers and enabling the patients to take care of their health [3]. The analytics helps to prevent patient suffering from the chronicle diseases .The use of analytics can improve accuracy, medical diagnostics earlier, enabling personalization and reducing the cost by avoiding lab procedures and also reduces the infection rate.IoT Healthcare is a combination of medical industry and IT industry.According to the survey released by IDC, the size of IOT healthcare market is expected to reach $\$ 12.4$ billion by 2018 .

Analytics on wearable sensors can make use ofpattern recognition and machine learning algorithms.The machine learning algorithm is used to create classification and helps for decision making of the disease[5]. The machine learning algorithm is defined as the adoption of computational methods for detection and describing consistencies and pattern from the trained data.

\section{HEALTHCARE ANALYTICS DATA SOURCES}

\section{A. Electronic Health Record}

Electronic Health Record are the digitized version of the people medical data. It provide people with medical care by sharing data with medical organization[4]. It also allows authorized medical user to edit records. The data are efficiently stored and retrieved leading to efficient decision support and quality management. They improve the diagnosis of diseases.

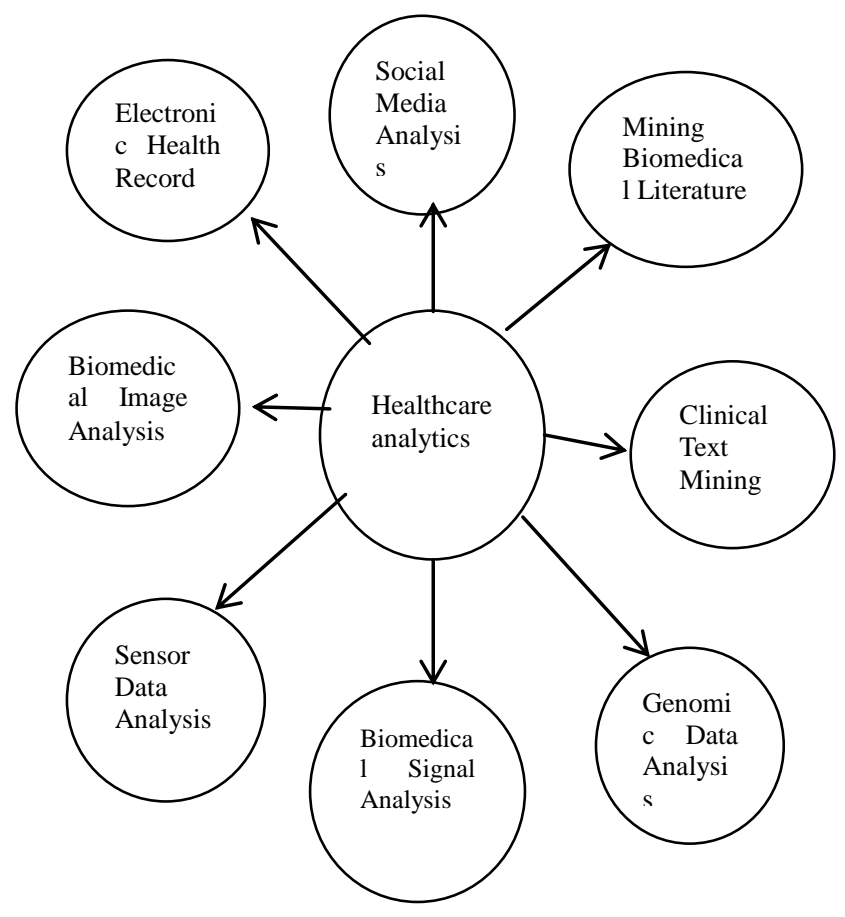

Fig.4. Healthcare analytics Data Sources

\section{B. Biomedical Image analysis}

Medical Image plays a vital role health care by providing anatomical structures of human beings[5]. Analyzing those images enables clinicians and researchers indulging disease monitoring and treatment planning. It generate quantitative information from the images.

\section{Sensor Data Analysis}

Sensors like Electrocardiogram, Electroencephalogram collect data from various parts of our body[6]. These are used for real time analytics which generate enormous amount of data leading to overloading challenge. These are managed by using analytic tools.

\section{Biomedical Signal Analysis}

Signals from electroneurogram (ENG), electrogastrogram (EGG), phonocardiogram (PCG) etc. are used for analyzing pathological conditions and helps in deciding an appropriate care pathway. The measurement of these signals gives perceptible assessment of the human body. These signals can be acquired invasively or non-invasively. Singular Value Decomposition (SVD), wavelet transformation and Principal Component Analysis (PCA) are some of the techniques used for analysis of these signals [24].

\section{E. Genomic Data Analysis.}

Most of the diseases are genetic in nature, but the relation between the genetic markers and the diseases has not been fully established. Translating genetic discoveries into personal medical care is a highly non-trivial task with a lot of technical challenges [7]. These challenges are overcomed by analytics techniques like Normalization and Quality Control, Differential Expression Detection, Clustering and Classification, Pathway and Gene Set Enrichment Analysis etc.

\section{F. Clinical Text Mining}

The information regarding patients are in the form of unstructured data format which form the backbone of healthcare. It becomes challenging to analyze automatically due to the complexity involved in converting unstructured data text to a structured format [8]. Natural Language Processing is thye major technique for obtaining knowledge from the unformatted text namely clinical data.

\section{G. Mining Biomedical Literature}

Text Mining Methods are used for preservation, availability and usability of digital resources in biomedical applications. They provide extracting , analyzing, and summarizing textual data leading researchers in knowledge discovery [21]. It benefits the biomedical research by linking textual evidence to biomedical pathways.

\section{H. Social Media Analysis}

The development of various social media resources provides significant information about people opinion on 
various aspects of healthcare. These are used for obtaining details regarding population health and public health monitoring. They focus on identifying aggregate health trends such as outburst of infectious diseases, tracking down reports on adverse drug interactions, and improving the health-related activities [22].

\section{MACHINE LEARNING ALGORITHMS}

The healthcare analytics uses various algorithms for identifying diseases from collected data. Machine learning algorithm provides an effective way for identification of diseases. Diseases like Heart disease, Liver diseases, Kidney diseases prediction can be done effectively by using machine learning algorithms by considering the data analytics.

There are variety of machine learning algorithms which acts effective for recognition of various diseases [11].

Machine learning algorithms are self learning algorithms which increases the accuracy of diagnosis by predictive analysis. They are classified as supervised machine learning algorithm and unsupervised machine learning algorithms. Supervised machine learning algorithms make prediction based on the trained set of samples. Whereas unsupervised machine learning algorithm organize data into clusters to describe its structure and draw inference from datasets of input data without labeled response [17].

\section{Supervised Machine Learning Algorithms}

Machine learning algorithms that makes prediction on given set of samples. It searches within the value labels assigned to datapoints.

Supervised algorithm consists of classification and regression. For example, classification predicts whether the patient will have a heart attack whereas a regression makes continuous measurement for an observation.

\section{Unsupervised Learning}

There are no labels associated with data points. These machine learning algorithm organize the data into a group of clusters to describe its design and make composite data look smooth and organized for analysis.

It is used in bioinformatics for sequence analysis and genetic clustering, Data mining for sequence and pattern mining, Medical imaging for image segmentation., Computer vision for object recognition.

\section{A. Decision Tree}

Decision tree are used to organize information in graphical form about possible options consequences and end value. They are predictive models used for simple decision making. The classification is done based on the arrangement of feature values [23].

\section{B. Naive Bayes Classification}

Naive Bayesian networks are made up of directed acyclic graphs where the parent representing the unobserved node and children representing the observed nodes. There is only one parent having several children and having independence between child nodes with respect to parents. Using the robust Bayes classifier increases the accuracy of prediction of diseases for intensive-care patients up to $78-84 \%$ [11].

\section{Bayesion Network Theory}

Bayesian networks also called the belief networks plays better role in the classification accuracy on diseases like Hepatitis, Pima Indian, Thyroid etc. [15] These are graphical models which represent knowledge about uncertain domain.

\section{Neural Network Based Learning}

Neural networks are classified into single layered neural networks and multilayered neural networks. Single layered neural network preserve maximum amount of information in the output unit[22].The hepatobiliary disorders can be found using the neural networks. Single layer neural network provide improvement in accuracy and speed. Multi layered neural network uses a supervised learning technique called the back propagation for training the set .

\section{E. Support Vector Machine}

SVM does classification of data by constructing an $\mathrm{N}$-dimensional hyper plane that separates into two categories. A group of features that define a cases called vector. Vectors near hyper plane are called as support vectors. Good separation is attained by largest distance separation to the nearest training data of any class. Larger the margin the lower is the generalization error. SVM algorithms are more suitable for identification of heart diseases. The table shows the comparison between various algorithms [11].

\section{DISCUSSION}

Machine learning algorithm is needed for classification of healthcare data set and for identification of diseases in an effective manner. There exists many algorithms for classification. The performance of algorithms are studied by considering the various parameters like efficiency, speed, classification ,rate of tolerance etc. The below table 1 shows the comparison among various algorithms by considering these parameters. Health data set classification seems more accurate by Supervised machine learning algorithm. 
Table 1. Comparison of Learning Algorithms.

\begin{tabular}{|l|c|c|c|c|c|}
\hline Parameters & $\begin{array}{c}\text { Decision } \\
\text { Trees }\end{array}$ & Rule Based & Naive Bayes & Neural Network & SVM \\
\hline Efficiency & Medium & Medium & Low & High & Very High \\
\hline Speed & High & Medium & Very High & Low & Medium \\
\hline Classification & High & Medium & High & Very High & Very High \\
\hline Event Attributes & High & Medium & Medium & Low & Very High \\
\hline Tolerance & Medium & Medium & Low & Medium & High \\
\hline Prevention & Medium & Medium & High & Low & Medium \\
\hline
\end{tabular}

Supervised machine learning algorithm works well in both linear and non-linear data sets. SVM are more suitable for identification of heart diseases, kidney disease, thyroid disease, diabetics etc. The SVM takes more running time but the accuracy rate in identification of diseases is high .SVM reduces the classification error and improves the performance rate.

In general SVM and neural networks perform better in continuous and multidimensional features. In SVM prediction accuracy is increased when large dataset is used. Naive Bayes classifier is less accurate on small data sets. With data that require diagonal partitioning decision tree algorithm does not perform well and it contains a complicated representation of data due to replication problem. It includes long running time. SVM performs well in data that contains different input and output features. It is very tolerant to irrelevant attributes. In general SVM performs better among the classifiers providing better accuracy. Next SVM comes, the neural networks are performing better. The table shows the comparison between various algorithms in terms of performance.

\section{CONCLusion}

Analytics in Internet of Things enables organization to collect and analyze data from sensors in environment providing better decision making in many situations, reducing maintenance cost etc., leading to development of technology. In the field of IoT healthcare, analytics improves the performance by efficiently identifying the diseases and provide better treatment to people. In this paper, we project the importance of Internet of Things in the healthcare industry. Continuous monitoring of patients using wearable sensors leads them to self care of their own health. The architectures used in IOT

Healthcare are also presented. Machine learning algorithms used in analytics helps to identify the diseases in efficient manner. Various machine learning algorithms for the classification of data's are also given.

\section{REFERNCES.}

[1] S. M. Riazul Islam, (member, ieee), Daehan Kwak, Md. Humaun kabir, Mahmud Hossain, and Kyung-sup kwak, (Member, IEEE), "The Internet of Things for Health Care: A Comprehensive Survey",June 4, 2015.

[2] Samuel, Robert E.; Connolly, Dennis , "Internet of things-based health monitoring and management domain-specific architecture pattern", Vol. 16 Issue 4, p58-63,2015.

[3] Amir-Mohammad Rahmani, Nanda Kumar Thanigaivelan, Tuan Nguyen Gia, Jose Granados, Behailu Negas, Pasi Liljeberg, and Hannu Tenhunen, "Smart e-Health Gateway: Bringing Intelligence to Internet-of-Things Based Ubiquitous Healthcare Systems",2015.

[4] Frederick J. Riggins, Samuel Fosso Wamba, "Research Directions on the Adoption, Usage, and Impact of the Internet of Things through the Use of Big Data Analytics", IEEE 30 March 2015.

[5] Tawseef Naqishbandi, Imthyaz Sheriff. C, Sama Qazi, "Big Data, CEP and IoT : Redefining Holistic Healthcare Information Systems and Analytics", International Journal of Engineering Research \& Technology Vol. 4 - Issue 01 (January - 2015).

[6] Soham Adhya; Dipak Saha ; Abhijit Das ; Joydip Jana ; Hiranmay Saha, "An IoT based smart solar photovoltaic remote monitoring and control unit Control, Instrumentation, Energy \& Communication (CIEC)", 2nd International Conference on 18 July 2016.

[7] Somayya Madakam, R. Ramaswamy, Siddharth Tripathi, "Internet of Things (IoT): A Literature Review,Journal of Computer and Communications", 164-173,2015.

[8] Ravi Teja Guthikonda, Sai Srikar Chitta , Shraddha Tekawade, Tripti Attavar, "Comparative Analysis Of IoT Architectures", April 25, 2014.

[9] Soumya Kanti Datta*, Christian Bonnet*, Amelie Gyrard**, Rui Pedro Ferreira da Costa*, Karima Boudaoud, "Applying Internet of Things for Personalized Healthcare in Smart Homes"20.

[10] Robert E. Samuel, Dennis Connolly, "Internet Of Things-Based Health Monitoring And Management Domain-Specific Architecture Pattern", Issues in Information Systems Volume 16, Issue IV, pp. 58-63, 2015. 
[11] S. Anto, Dr.S.Chandramathi, "Supervised Machine Learning Approaches for Medical Data Set Classification A Review", IJCST Vol. 2, Iss ue 4, Oct . - Dec. 2011

[12] Luigi Atzori a, Antonio Iera b, Giacomo Morabito, "The Internet of Things: A survey", Computer Networks 54 (2010) 2787-2805.

[13] Shancang Li \& Li Da Xu \& Shanshan Zhao, The internet of things: A survey, 26 April 2014

[14] Moeen Hassanalieragh, Alex Page, Tolga Soyata, Gaurav Sharma, Mehmet Aktas, Gonzalo Mateos, Burak Kantarci, Silvana Andreescu, "Health Monitoring and Management Using Internet-of-Things (IoT) Sensing with Cloud-based Processing: Opportunities and Challenges", IEEE International Conference on Services Computing,2015.

[15] Melanie Swan "The Internet of Things, Wearable Computing, Objective Metrics, and the Quantified Self 2.0, J. Sens. Actuator Netw. 1, 217-253,2012.

[16] F'elix Jes'us Villanueva, David Villa, Francisco Moya, Maria Jos'e Santofimia, Juan Carlos L'opez, "Internet of Things architecture for a RFID-based product tracking business model".

[17] N. Saravanan, Dr. N. Preethi , "Survey on Analytics, Machine Learning and the Internet of Things Computer Technology \& Applications",Vol 6 (6),1042-1046 .

[18] S.Sivagami,D.Revathy, L.Nithyabharathi, "Smart Health Care System Implemented Using IoT" ,International Journal of Contemporary Research in Computer Science and Technology (IJCRCST) $e$-ISSN: 2395-5325 Volume 2, Issue 3 (March '2016).

[19] $\mathrm{Wu} \mathrm{He}$,Shancang Li , "Internet of Things in Industries: A Survey IEEE Transactions on Industrial Informatics", November 2014.

[20] Shashank Agrawal, Dario Vieira, "A survey on Internet of Things”,ISSN:2316-9451 ,v. 1, n. 2, p. 78 - 95(2013).

[21] G. Zhang, C. Li, Y. Zhang, C. Xing, and J. Yang, "Seman Medical: A kind of semantic medical monitoring system model based on the IoT sensors", in Proc. IEEE Int. Conf. eHealth Netw., Appl. Services (Healthcom), Oct. 2012.

[22] Z. Jian, W. Zhanli, and M. Zhuang, "Temperature measurement system and method based on home gateway", Chinese Patent 102811185 A, Dec. 5, 2012.

[23] L. Yang, Y. Ge, W. Li, W. Rao, and W. Shen, "A home mobile healthcare system for wheelchair users", in Proc. IEEE Int. Conf. Comput. Supported Cooperat. Work Design (CSCWD), pp. 609614,May 2014.
[24] Jasper Tan, Simon G. M. Koo, “A Survey of Technologies in Internet of Things", IEEE International Conference on Distributed Computing in Sensor Systems 2014.

\section{Authors' Profiles}

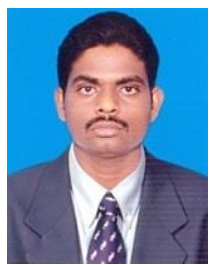

G.Shanmugasundaram obtained his B.Tech in Information Technology (2005) from BCET, Pondicherry university. $\mathrm{He}$ received his M.Tech in Computer Science and Engineering (2008) from SMVEC, Pondicherry University. He was awarded Doctorate from Pondicherry University (2013). Currently he is working Assistant Professor at SMVEC, Pondicherry. He is having 6 years of teaching experience and 1 year in industry. He has published several papers in national and international journals/ conferences. His research area includes Service Oriented Architecture, Service Discoverability, Software Metrics and Web Technologies.

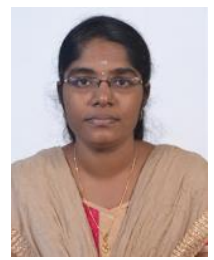

G.SankariKaarguzhali obtained her B.Tech in Electronics and communication engineering(2015) from Christ College of Engineering and Technology, Pondicherry.Currently she is persuing M.Tech in Networking at SMVEC,Pondicherry Univeristy.Her Research areas include Image Processing, Semantic Web.

How to cite this paper: Shanmugasundaram.G, Sankarikaarguzhali.G,"An Investigation on IoT Healthcare Analytics", International Journal of Information Engineering and Electronic Business(IJIEEB), Vol.9, No.2, pp.11-19, 2017. DOI: 10.5815/ijieeb.2017.02.02 